\title{
A Novel Approach to Route Selection in Car Navigation Systems by a Multiobjective Genetic Algorithm
}

\author{
Feng Wen \\ Graduate School of Information, \\ Production \& Systems, \\ Waseda University \\ Kitakyushu, 808-0135, Japan \\ 81(93)692-5273 \\ fengwen@ruri.waseda.jp
}

\author{
Xiaohao Gao \\ Graduate School of Information, \\ Production \& Systems, \\ Waseda University \\ Kitakyushu, 808-0135, Japan \\ 81(93)692-5273 \\ gao@fuji.waseda.jp
}

\author{
Mitsuo Gen \\ Graduate School of Information, \\ Production \& Systems, \\ Waseda University \\ Kitakyushu, 808-0135, Japan \\ 81(93)692-5273 \\ gen@waseda.jp
}

\begin{abstract}
This paper considers the multicriteria route selection problem (mRSP) for car navigation systems in traffic road network. A multi-layer hierarchy network method is proposed to substantially reduce the computation time when solving mRSP. We also propose a new multiobjective Genetic Algorithm (moGA) named distance-based nondominated sorting Genetic Algorithm (dnsGA) to generate evolutionary results for mRSP. It attains better convergence to the Pareto-optimal front and gives sufficient emphasis to the diversity consideration. The experiment results showed the effectiveness and the efficiency of our approach.
\end{abstract}

\section{Categories and Subject Descriptors}

I.2.8 [ARTIFICIAL INTELLIGENCE]: Problem Solving, Control Methods, and Search - Heuristic methods

\section{General Terms}

Algorithms

\section{Keywords}

Intelligent Transport System (ITS); multicriterion route selection problem (mRSP); multiobjective genetic algorithm (moGA).

\section{INTRODUCTION}

Car navigation systems (CNS) are the most widely used form of information terminals for the Intelligent Transport Systems (ITS). Route selection (RS) is the most important function for CNS. Route selection problems (RSP) in CNS are search problems for finding an optimal route from an original point to a destination point on a road network. It is a multicriteria route selection problem (mRSP). Genetic algorithms (GA) are have received considerable attention regarding their potential as a novel approach to multiobjective optimization problems, known as evolutionary or genetic multiobjective optimization [1][2]. In this paper, we propose a multi-layer hierarchy network method that can substantially reduce the route computation time and select a more practical route for driver. On the other hand, a novel elitist multiobjective genetic algorithm, called distance-based nondominated sorting Genetic Algorithm (dnsGA), is presented to effectively solve multicriteria problem. We consider objectives

Copyright is held by the author/owner(s).

GECCO'08, July 12-16, 2008, Atlanta, Georgia, USA.

ACM 978-1-60558-130-9/08/07. include minimizing driving distance, minimizing driving time and minimizing driving cost. We denote some parameters here, $n$ : number of nodes; $\boldsymbol{p}$ : a selected route from origin $O$ to destination $D ; d_{i j}$ : the length of link $(i, j) ; l_{i j}$ : the number of lanes on link $(i, j)$; $v_{i j}$ : the speed limit on link $(i, j) ; c_{i j}$ : the toll fee on link $(i, j)$;

objective function for minimizing driving distance on a route $\boldsymbol{p}$ :

$$
\min f_{1}(\boldsymbol{p})=\sum_{(i, j) \in p} d_{i j} x_{i j}
$$

where $(i, j)$ is links from node $i$ to node $j$ in link set $A$, $d_{i j}$ is the length of a link

The objective function for minimizing driving time on a route $\boldsymbol{p}$ :

$$
\min f_{2}(\boldsymbol{p})=\sum_{(i, j) \in \boldsymbol{p}} g\left(d_{i j}, v_{i j}\right) x_{i j}
$$

The function for minimizing driving cost on a route $\boldsymbol{p}$ :

$$
\min f_{3}(\boldsymbol{p})=\sum_{(i, j) \in p} h\left(d_{i j}, c_{i j}\right) x_{i j}
$$

\section{HIERARCHY NETWORK METHOD}

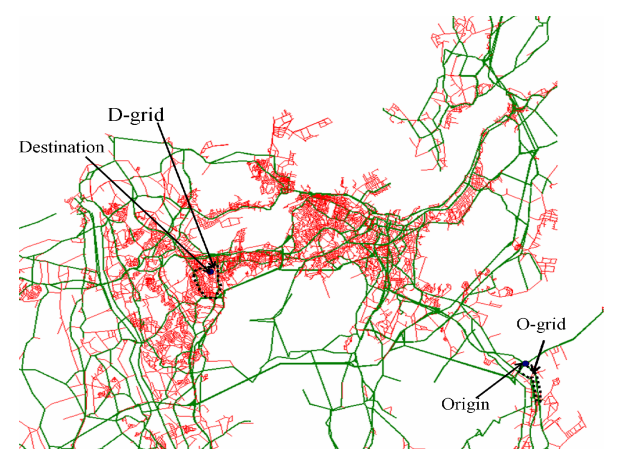

Figure 1. The real road map of Kitakyushu city in Japan.

The major roads partition the whole network into many smaller sub networks. These sub networks are referred to as grids. A grid includes a collection of minor roads bounded on all sides by major roads. The grids containing the origin $O$ and the destination $D$ are named $O$-grid and $D$-grid. The new network created by 
hierarchy network method is composed of the three parts of components (see Figure 1):

1. All links belonged major road and all intersection nodes of major road link. This part is denote as $G_{\mathrm{h}}$;

2. In $O$-grid, all links and all intersection nodes. This part is denote as $G_{\mathrm{O}}$;

3. In $D$-grid, all links and all intersection nodes. This part is denote as $G_{\mathrm{D}}$;

The road network of Kitakyushu in Japan is shown in Figure 1. There are 16175 nodes and 24238 links in this digital road map. There are 345 nodes and 582 links in $G_{\mathrm{h}}, G_{\mathrm{O}}$ and $G_{\mathrm{D}}$.

\section{DISTANCE-BASED NONDOMINATED SORTING GENETIC ALGORITHM}

\subsection{Pareto Distance}

We use $E_{t}$ to denote the current Pareto solution set in generation $t$. Firstly, each individual $\boldsymbol{x}$ of current population $P(\mathrm{t})$ and offspring $C(\mathrm{t})$ should calculate a distance to $E_{t}$. For unification, all objective are assumed to be minimized for clarity purposes. To avoid a scale problem within the objectives, we should normalize the objective value. When considering the minimization problem with $q$ objectives, we define two extreme points: the maximum extreme point $z^{+}=\left\{z_{1}{ }^{\max }, z_{2}{ }^{\max }, \ldots, z_{q}{ }^{\max }\right\}$ in solution area in each generation. And the minimum extreme point $e^{-}=\left\{e_{1}{ }^{\min }, e_{2}{ }^{\min }, \ldots\right.$, $\left.e_{q}{ }^{\min }\right\}$ in $E_{t}$ of each generation. We denote $d_{\mathrm{P}}$ as this distance:

$$
d_{\mathrm{p}}(\boldsymbol{x})=\min \left\{\sqrt[2]{\sum_{m=1}^{q}\left(\left(f_{m}(\boldsymbol{x})-f_{m}(\boldsymbol{r})\right) /\left(z_{m}^{\max }-e_{m}^{\min }\right)\right)^{2}} \mid \boldsymbol{r} \in E_{t}\right\},
$$

here, $\boldsymbol{x}$ is current individual in $P(t)$ and $C(t), \boldsymbol{r}$ is element in $E_{t}$.

\subsection{Density distance}

In this paper, the crowding distance procedure is based on nsGAII. We denote $d_{\mathrm{C}}$ as crowding distance.

\subsection{Distance-based Nondominated Sorting}

We use these two distance value as factor objective values of each individual. And the factor objective values $\left(d_{\mathrm{P}}\right.$ and $\left.d_{\mathrm{C}}\right)$ are used as substitute of origin objective values. The multiobjective problem with multicriteria is treated as two objectives. And the objectives are minimizing $d_{\mathrm{P}}$ and maximizing $d_{\mathrm{C}}$. When select the next generation, we use nondominated sorting method to decide which individual is selected. The nondominated sorting procedure can be found in nsGA-II. The Genetic operator is standard method.

\section{COMPUTATIONAL EXPERIMENTS AND DISSCUSSIONS}

In this section, we present empirical results for evaluating the computational performance of the proposed multi-layer hierarchy network method and dnsGA. Our algorithm is coded in Java and run on a $3.2 \mathrm{GHz}$ Pentium with 3-GB RAM. The computational evaluation is based on digital road maps of Kitakyushu city. We evaluate proposed algorithms through computational experiments under bellowed GA parameter settings: population size, popSize $=$ 20 ; crossover probability, $p_{\mathrm{C}}=0.70$; mutation probability, $p_{\mathrm{M}}=$ 0.60 ; stopping condition, $\operatorname{maxGen}=2000$. Each simulation is running 20 times. The Pareto solution set of experiment case one is shown in Figure 2.

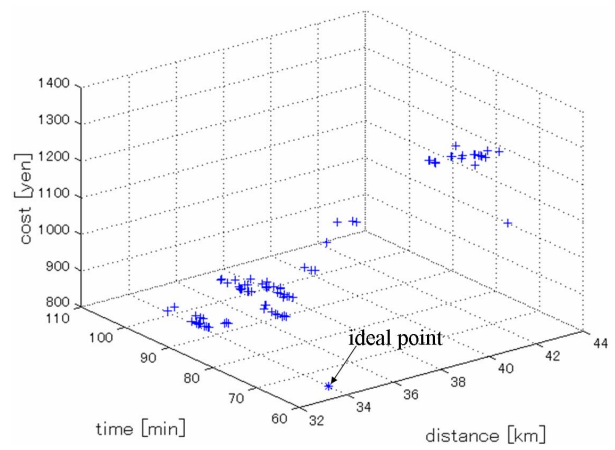

Figure 2. Solution result.

Here we use $w_{\mathrm{L}}, w_{\mathrm{T}}, w_{\mathrm{C}}$ as factor weight for route length objective, time objective, cost objective respectively, and $w_{\mathrm{L}}+w_{\mathrm{T}}$ $+w_{\mathrm{C}}=1$. We denote $w_{r l}$ as the weight factor:

$$
w_{r l}=\sqrt{w_{\mathrm{L}}\left(\frac{f_{1}(\boldsymbol{x})-f_{1}(\boldsymbol{l})}{z_{1}{ }^{\max }-f_{1}(\boldsymbol{l})}\right)^{2}+w_{\mathrm{T}}\left(\frac{f_{2}(\boldsymbol{x})-f_{2}(\boldsymbol{l})}{z_{2}{ }^{\max }-f_{2}(\boldsymbol{l})}\right)^{2}+w_{\mathrm{C}}\left(\frac{f_{3}(\boldsymbol{x})-f_{3}(\boldsymbol{l})}{z_{3}{ }^{\max }-f_{3}(\boldsymbol{l})}\right)^{2}},
$$

Table 1. Best compromised solution

\begin{tabular}{|l|l|l|c|}
\hline & $\begin{array}{l}f_{\mathbf{1}}(\boldsymbol{x}): \text { Length } \\
{[\mathbf{m}]}\end{array}$ & $\begin{array}{l}\boldsymbol{f}_{\mathbf{2}}(\boldsymbol{x}) \text { : Time } \\
{[\mathrm{min}]}\end{array}$ & $\begin{array}{l}\boldsymbol{f}_{\mathbf{3}}(\boldsymbol{x}) \text { :Cost } \\
{[\text { yen] }}\end{array}$ \\
\hline $\begin{array}{l}w_{\mathrm{L}}=w_{\mathrm{T}}=w_{\mathrm{C}} \\
=1 / 3\end{array}$ & 39335 & 78.9 & 1191.2 \\
\hline $\begin{array}{l}w_{\mathrm{L}}=1, w_{\mathrm{T}}= \\
w_{\mathrm{C}}=0\end{array}$ & 33862 & 87.7 & 956.2 \\
\hline $\begin{array}{l}w_{\mathrm{L}}=0, w_{\mathrm{T}}= \\
1, w_{\mathrm{C}}=0\end{array}$ & 41101 & 61.1 & 1383.6 \\
\hline
\end{tabular}

\section{CONCLUSION}

In this paper, we also proposed distance Pareto Genetic Algorithm (dnsGA) to ensure the Pareto solution set of dnsGA own better convergence and diversity features. The effectiveness and efficiency of dnsGA was investigated with different scales of road map by comparing with recent related researches.

\section{ACKNOWLEDGMENTS}

The authors are grateful for the financial support received from the JSPS \& Toyota Motors for this research project. The author is also deeply indebted to the Japan Digital Road Map Association for providing the digital road-map database.

\section{REFERENCES}

[1] Gen, M. and Cheng, R., 2000. Genetic Algorithms and Engineering Optimization. Wiley, New York..

[2] Gen, M., Cheng, R. and Oren, S.S., 2001. Network Design Techniques using Adapted Genetic Algorithms. Advances in Engineering Software 32 (9), 731-744. 